\title{
Biopsy Findings in Renal Allograft Dysfunction in a Live Related Renal Transplant Program
}

\section{Javed I Kazi and Muhammed Mubarak*}

Department of Histopathology, Sindh Institute of Urology and Transplantation, Karachi

\author{
Abstract \\ Background: There is little information in literature on renal allograft biopsy findings in renal allograft dysfunction \\ in live related renal transplant recipients.
}

Material and Methods: A retrospective review of 1210 renal allograft biopsies from 575 renal transplant patients was carried out over a period of seven years from June 1997 till December 2004. The demographic, clinical, laboratory and biopsy findings were collected and analyzed.

Results: A total of 1210 graft biopsies were performed on 575 patients. The mean age of recipients and donors was $29.2 \pm 9.7$ years, and $35.7 \pm 10.5$ years, respectively. The males were predominant among recipients (76.7 vs. $23.3 \%$ ), while among donors they only slightly outnumbered females (51.8 vs. $48.2 \%)$.

Regarding pathological lesions, acute rejection was seen in $292(24 \%)$ cases, followed by acute tubular injury and cyclosporine A (CsA) toxicity, found in $281(23.2 \%)$ and $134(11 \%)$ cases respectively. Chronic allograft nephropathy (CAN) with variable degree of tubular atrophy was seen in $361(29.8 \%)$ cases. Seventy nine cases $(6.5 \%)$ of acute pyelonephritis were detected on graft biopsies. A number of rare lesions were also found, including $13(1.07 \%)$ cases of recurrent/de novo renal disease, and $13(1.07 \%)$ of polyoma virus infection. Five cases of CsA induced hemolytic uremic syndrome (HUS) were also noted.

Conclusion: In conclusion, the incidence of acute rejection is low in our patients as compared to cadaveric renal transplant recipients as reported in Western studies and CsA toxicity is more common. Recurrent/de novo renal disease is uncommon in our patients.

Keywords: Allograft biopsy; Immunosuppression; Renal transplantation; Acute rejection; Cyclosporine toxicity

\section{Introduction}

Renal transplantation is the treatment modality of choice for patients with end-stage renal disease (ESRD) throughout the world [1]. The short-term renal transplant outcome has improved markedly during the last few decades due to improved surgical techniques, better medical care, prevention and treatment of infections, but above all, due to advancements in the field of immunosuppressive treatment [1]. Despite the above accomplishments, renal allograft dysfunction is still common after transplantation and may be caused by acute rejection, chronic rejection, calcineurin inhibitor (CNI) toxicity, infections and other rare causes such as recurrence of original renal disease. Each of the above causes of renal allograft dysfunction requires different therapeutic approach and hence accurate diagnosis is essential for the optimal management of the patients [2]. Clinical diagnosis is unreliable as shown by several studies reporting inability to accurately predict the cause of graft dysfunction in 40 to $70 \%$ of cases based on the clinical criteria alone [3-7]. Renal allograft biopsy is the gold standard to accurately establish the cause of renal allograft dysfunction $[3,8,9]$. It is generally felt that the causes of graft dysfunction vary in live related vs. cadaveric renal transplant settings as well as in different immunosuppressive protocols [10-21]. There are also center to center, and inter-institutional variations in the quality and the incidence of rejection [22-26]. There are very few studies exclusively on the causes of graft dysfunction in a live related renal transplant program [10-15].

The estimated incidence of ESRD in Pakistan is 100 per million population (pmp), with approximately 18,000 new cases each year. More than $90 \%$ of the ESRD population in this country is disfranchised from renal replacement therapy (RRT), 10\% receive dialysis and $4-5 \%$ receive transplants at a rate of $<5 \mathrm{pmp}$ [16].

We undertook this preliminary study to evaluate the causes of renal graft dysfunction as detected on renal allograft biopsies in a fairly large cohort of live related renal transplant patients and to compare our findings with those in the literature.

\section{Materials and Methods}

A retrospective review of 1210 biopsies from 575 renal transplant patients was carried out over a period of seven years from June 1997 till December 2004. Following data items were collected from a review of original renal allograft biopsy request forms and clinical charts; demographics of recipients and donors, donor source and relation, human leukocyte antigen (HLA match), and allograft biopsy findings. Renal allograft biopsies were performed when there was unexplained graft dysfunction (rise in serum creatinine of $\geq 20 \%$ over baseline) and/ or proteinuria, fulfilling the established indications of graft biopsies $[26,27]$. Two cores of renal graft tissue are obtained with automated biopsy gun under real-time ultrasound guidance. In cases with proteinuria or strong clinical suspicion for acute humoral rejection (AHR), an additional core was obtained for immunofluorescene, C4d, and electron microscopy (EM) [28]. The cores for light microscopy (LM) were fixed in $10 \%$ buffered formalin and processed for paraffin

*Corresponding author: Dr. Muhammed Mubarak, Associate professor, Department of Histopathology, Sindh Institute of Urology and Transplantation, Karachi-74200, Pakistan, Tel: 009221 99215752; Fax: 009221 32726165; E-mail: drmubaraksiut@yahoo.com

Received September 26, 2011; Accepted October 31, 2011; Published November 03, 2011

Citation: Kazi Jl, Mubarak M (2012) Biopsy Findings in Renal Allograft Dysfunction in a Live Related Renal Transplant Program. J Transplant Technol Res 2:108. doi:10.4172/2161-0991.1000108

Copyright: (c) 2012 Kazi JI, et al. This is an open-access article distributed under the terms of the Creative Commons Attribution License, which permits unrestricted use, distribution, and reproduction in any medium, provided the original author and source are credited. 
embedding. The biopsies are processed and reported on the same day with urgent processing and preparation of the tissue, and appropriate management instituted. A set of ten slides with multiple serial sections were cut and stained with hematoxylin and eosin (H\&E), periodic acid-Schiff reagent (PAS), trichrome and silver stains as recommended in Banff schema [27]. The histological changes were interpreted and classified according to Banff 97 working classification of renal allograft pathology [27].

\section{Immunosuppression protocol}

The immunosuppressive drugs were started a day before transplantation and all patients received standard triple drug immunosuppression in standard dosages: cyclosporine/FK506, azathioprine/mycophenolate mofetil (MMF), and steroids [10].

Patients with high panel reactive antibodies (PRA) or cadaveric transplants, received induction therapy with antithymocyte/ antilymphocyte globulin while other high-risk groups (second transplant, spousal transplants, historical high PRA) received Interleukin-2 (IL-2) receptor blockers.

\section{Statistical analysis}

Statistical analysis was carried out using IBM compatible SPSS for Windows version 10 (SPSS, Chicago, IL, USA). Simple descriptive statistics such as mean \pm standard deviation (SD) were used for continuous variables such as age and clinical and laboratory parameters. Numbers (percentages) were used for categorical data, such as biopsy diagnoses.

\section{Results}

A total of 1210 graft biopsies were performed in 575 patients from June 1997 to December 2004 at a rate of 2.1 biopsies per patient. The demographic data of the recipients, the donors and the donor-recipient relationship are given in Tables 1, and 2 respectively. The renal allograft recipients were relatively younger than donors with a mean age of $29.2 \pm 9.7$ years. For donors, the mean age was $35.7 \pm 10.5$ years. The gender distribution of the recipients and donors is also provided in Table 1 and shows a vast preponderance of males in recipients.

On HLA matching, a majority of recipients (70.8\%) showed at least one haplotype match, $10.8 \%$ showed less than one haplotype antigen match, and $17.7 \%$ were HLA identical with the donors. Regarding pathological lesions, the overall distribution of the various diagnoses is shown in Table 3. Acute rejection (AR) was seen in $292(24 \%)$ cases. A breakdown of the different types of rejection is provided in Table 4 , which shows that type I or tubulointerstitial rejection was the most common type of rejection, and most of these cases were mild (IA). AR was followed by acute tubular injury and cyclosporine A (CsA) toxicity, found in $281(23.2 \%)$ and $134(11 \%)$ cases, respectively. Most cases of acute tubular injury were mild in nature and most probably resulted from the toxic effects of CsA rather than being ischemic in origin, as most of them improved on dose reduction of cyclosporine. Taking together both the above lesions, CsA constituted the largest single cause of graft dysfunction in our patients. Interstitial fibrosis/tubular atrophy (IFTA) with variable degree of tubular atrophy was seen in 361 (29.8\%) cases. Seventy nine cases $(6.5 \%)$ of acute pyelonephritis were detected in graft biopsy. Majority of these cases were first picked up on biopsy with no clinical suspicion of infection. A number of rare lesions were found in $29(2.4 \%)$ cases. These included 13 cases of recurrent/ de novo renal disease, as shown in Table 5. Five cases of CsA induced hemolytic uremic syndrome (HUS) were also noted in the series. These were mild in nature and characterized by fibrin thrombi in the glomerular capillaries. These reverted to normal upon withdrawal of immunosuppression.

\section{Discussion}

This is one of the largest studies in the literature on the spectrum of pathological changes seen on renal allograft biopsies in live related renal transplant patients from a single center in Pakistan. The findings from this study are an important contribution to the existing sparse literature on this subject, especially in the context of the live related renal transplant program.

The demographic profile of the recipients and donors is more or less similar to that reported from neighboring country $[10,11]$. However, the donor source in nearly all of our cases was live related. In one Indian study, more than $70 \%$ of donors were live related (LRD) and the remaining were live unrelated (LURD) and cadaveric sources [10]. Another Indian study also reported almost half of transplants from LRDs and remaining from LURDs [11].

Acute rejection (AR) is the most dreaded complication of any allograft transplant and the most frequent clinical question for which allograft biopsies are performed. It was found in $24 \%$ of our patients. This is low as compared with the prevalence of around $40 \%$ noted in the earlier studies and studies on the cadaveric transplants [6,7]. However, the rates are comparable to those found in other living related renal transplant studies $[10,11]$. In the Indian study, acute rejection was observed in $27.3 \%$ of cases [10]. In the majority of our cases, AR was of the tubulo-interstitial or cellular type, of milder phenotype, and belonged to IA category of Banff classification, as shown in Table $4[27,28]$. In a small but significant number of cases $(11.6 \%)$, the rejection process was of the borderline category according to Banff 97 classification. Almost all of these cases were treated with methyl prednisolone pulse and responded with a decline of serum creatinine to pretreatment levels (unpublished data). Both the above findings may be the result of a highly pro-active renal allograft biopsy approach adopted at our center. The diagnosis of early acute cellular rejection is often challenging and may be missed if Banff criteria are applied [22-25,29]. Use of a computer-based artificial neural network such as Bayesian

\begin{tabular}{|l|l|l|l|}
\hline & Males & Females & Mean age in years (range) \\
\hline Recipients & $441(76.7 \%)$ & $134(23.3 \%)$ & $29.2 \pm 9.7(10-57)$ \\
\hline Donors & $298(51.8 \%)$ & $277(48.2 \%)$ & $35.7 \pm 10.5(18-68)$ \\
\hline
\end{tabular}

Table 1: The demographic data of 575 recipients, and the donors.

\begin{tabular}{|l|l|l|}
\hline Relationship & Number & Percentage \\
\hline Siblings & 289 & $50.3 \%$ \\
\hline Parents & 173 & $30.1 \%$ \\
\hline Others & 113 & $19.6 \%$ \\
\hline Total & 575 & 100 \\
\hline
\end{tabular}

Table 2: Donor relationship with the recipients $(n=575)$.

\begin{tabular}{|l|l|l|}
\hline Pathologic lesions & Number & Percentage \\
\hline Active / acute rejection & 292 & 24.1 \\
\hline Cyclosporine toxicity & 134 & 11 \\
\hline Acute tubular injury & 281 & 23.2 \\
\hline Acute pyelonephritis & 79 & 6.5 \\
\hline Interstitial fibrosis/tubular atrophy & 361 & 29.8 \\
\hline No significant pathology & 34 & 2.8 \\
\hline Others & 29 & 2.4 \\
\hline Total & $\mathbf{1 2 1 0}$ & $\mathbf{1 0 0}$ \\
\hline
\end{tabular}

Table 3: Major categories of pathological lesions on 1210 renal allograft biopsies from 575 patients. 
Belief Network (BBN) approach using multiple pathology variables has been shown to increase the accuracy of diagnosis of early acute cellular rejection [22-25]. The diagnosis of acute vascular rejection (AVR) is often straight forward. This type of rejection was less common in our series, being detected in approximately one fifth of AR cases. Similar to cellular rejection, AVR was also mild in the majority of cases. Most cases of AVR belonged to IIA or IIB category. Very few cases of type III or transmural AVR were noted, as shown in Table 4. Acute humoral rejection (AHR) was distinctly uncommon in our patients, found in only three cases, which is not surprising given the live related nature of the donors, and first transplants in the vast preponderance of cases in our cohort [28]. The diagnosis of AHR is made according to revised Banff classification taking into account the morphology, C4d positivity and the detection of donor specific antibody by flow cross match [29].

CNI toxicity was also a major cause of allograft dysfunction in the acute setting in our patients. The diagnosis of this complication is often difficult and challenging $[2,15,16-19]$. The toxicity of the drugs may be caused in the face of normal or even low levels of the drug in the blood. Most of the morphological changes of CNI toxicity are non-specific except for the nodular arteriolar hyalinosis [2]. In many cases, acute tubular injury coupled with dystrophic calcification and isometric vacuolization of tubular epithelial cells was the only evidence of $\mathrm{CNI}$ toxicity.

In the long term, the most common cause of graft dysfunction was interstitial fibrosis/tubular atrophy, not otherwise specified (IFTA, NOS). Indeed, the prevalence of this diagnosis in the renal allograft biopsies increases with the prolongation of posttransplant period, as reported by other investigators [30-32]. In majority of the cases, IFTA was of no specific etiology, which is similar to all the previously published studies on this subject [30-32]. IFTA is not a single entity but rather a designation for the chronic changes affecting the parenchyma and may be caused by a large number of injurious agents. Previously IFTA was designated as chronic allograft nephropathy (CAN). The later designation was replaced by a more descriptive term of interstitial fibrosis/tubular atrophy (IF/FA), with a qualifier NOS (not otherwise specified) if no underlying cause is identified in the biopsy material in Banff '05 update [32].

We also observed a few cases of acute pyelonephritis of bacterial origin on renal allograft biopsies. The urine culture did not reveal bacterial growth in majority of these cases. This is interesting observation and has been reported previously [33]. Probably the infection is of non-communicating type. Among viral causes, polyoma virus was detected in 13 cases. The polyoma virus infection was confirmed by quantitative polymerase chain reaction (PCR) of the urine and serum. This opportunistic infection has emerged as an important cause of renal allograft dysfunction and graft loss in recent years [34]. Other less common causes of renal allograft dysfunction included 13 cases of recurrent/de novo glomerular diseases. A breakdown of these lesions is given in Table 5. The incidence of these lesions also increases with the prolongation of post-transplant duration [35]. In a significant minority of cases, no obvious pathology was detected on allograft biopsies. This may have resulted from inherent sampling error or some other non-parenchymal cause for the graft dysfunction. However, in an overwhelming majority of cases, the renal allograft biopsy was of immense help in the correct diagnosis and management of renal transplant patients.

In conclusion, the study defines the causes of graft dysfunction as detected on indicated graft biopsies in a large cohort of live related renal transplant patients. The incidence of acute rejection is low in

\begin{tabular}{|l|l|l|}
\hline Types of rejection & Numbers & Percentage \\
\hline Antibody mediated rejection & 3 & 1 \\
\hline Borderline rejection & 34 & 11.6 \\
\hline Tubulointerstitial rejection & 198 & 67.8 \\
\hline IA & 169 & 57.8 \\
IB & 29 & 9.9 \\
\hline Vascular rejection & 57 & 19.5 \\
IIA & 43 & 14.7 \\
IIB & 10 & 3.4 \\
III & 4 & 1.4 \\
\hline
\end{tabular}

Table 4: Breakdown of the 292 cases of acute/active rejection according to Banff classification.

\begin{tabular}{|l|l|l|}
\hline Pathological lesions & Number & Percentage \\
\hline Focal segmental glomerulosclerosis & 5 & 0.4 \\
\hline IgA nephropathy & 3 & 0.2 \\
\hline Mesangiocapillary GN-I & 2 & 0.1 \\
\hline Focal necrotizing GN & 2 & 0.1 \\
\hline Fabry's disease & 1 & 0.08 \\
\hline Total & 13 & 1.07 \\
\hline
\end{tabular}

Table 5: Recurrent/de novo renal diseases on 1210 renal allograft biopsies in 575 patients.

our patients as compared to cadaveric renal transplant recipients and CsA toxicity is more common. Recurrent/de novo renal disease is uncommon in our patients.

\section{References}

1. Hariharan S, Johnson CP, Bresnahan BA, Taranto SE, Mcintosh MJ, et al. (2000) Improved graft survival after renal transplantation in United States 1988-1996. N Eng J Med 342: 605-612.

2. Colvin RB (1996) The renal allograft biopsy. Kidney Int 50: 1069-1082.

3. Al-Awwa I, Hariharan S, First MR (1998) The importance of allograft biopsy in renal transplant recipients: correlation between clinical and histological diagnosis. Am J Kidney Dis 31: S15-S18.

4. Gaber L (1998) Role of renal allograft biopsy in multicentre clinical trials in transplantation. Am J Kidney Dis 31: S19-S25.

5. Parfrey P, Kuo Y, Hanley J, Knaack J, Xue Z, et al. (1984) The diagnostic and prognostic value of renal allograft biopsy. Transplantation 38: 586-590.

6. Matas AJ, Sibley R, Mauer M, Sutherland DE, Simmons RL, et al. (1983) The value of needle renal allograft biopsy. I. A retrospective study of biopsies performed during putative rejection episodes. Ann Surg 197: 226-237.

7. Matas AJ, Tellis VA, Sablay L, Quinn T, Soberman R, et al. (1985) The value of needle renal allograft biopsy. III. A prospective study. Surgery 98: 922-926.

8. Akhtar F, Rana TA, Kazi J, Zafar N, Hashmi A, et al. (1998) Correlation between biopsies and noninvasive assessment of acute graft dysfunction. Transplant Proc 30: 3069.

9. Wilckzek HE (1990) Percutaneous needle biopsy of the renal allograft. A safety evaluation of 1129 biopsies. Transplantation 50: 790-797.

10. Verma PP, Hooda AK, Sinha T, Chopra GS, Karan SC, et al. (2007) Renal transplantation- an experience of 500 patients. MJAFI 63: 107-111.

11. Mishra MN, Saxena VK, Narula AS (2004) Differences in renal transplantation in India and first world countries. Int J Hum Genet 4: 161-165.

12. D'Alessandro AM, Sollinger HW, Knechtle SJ, Kalayoglu M, Kisken WA, et al (1995) Living related and unrelated donors for kidney transplantation- a 28-year experience. Ann Surg 222: 353-364.

13. Matas AJ, Payne WD, Sutherland DE, Humar A, Greessner RW, et al. (2001) 2,500 living donor kidney transplants: a single-center experience. Ann Surg 234: 149-164.

14. Ratnakar KS, George S, Datta BN, Fayek AH, Rajagopalan S, et al. (2002) Renal transplant pathology: Bahrain experience. Saudi J Kidney Dis Transplant 13: 71-76. 
15. Mazzali M, Ribeiro-alves MA, Alves FG (1999) Percutaneous renal graft biopsy: a clinical, laboratory and pathological analysis. Sao Paulo Med J 117: 57-62.

16. Rizvi SAH, Naqvi SAA, Zafar MN, Hussain Z, Hashmi A, et al. (2011) A renal transplantation model for developing countries. Am J Transplant 11: 23022307.

17. Myers BD, Ross J, Newton L, Luetscher J, Perlroth M (1984) Cyclosporineassociated chronic nephropathy. N Engl J Med 311: 699-705.

18. Mihatsch MJ, Thiel G, Basler V, Ryffel B, Landmann J, et al. (1985) Morphological patterns in cyclosporine-treated renal transplant recipients. Transplant Proc 17: 101-116.

19. Pascual M, Vallhonrat $H$, Cosimi AB, Tolkoff-Rubin N, Colvin RB, et al. (1999) The clinical usefulness of the renal allograft biopsy in the cyclosporine era: a prospective study. Transplantation 67: 737-741.

20. Bergstrand A, Bohman SO, Farnsworth A (1985) Renal histopathology in kidney transplant recipients immunosuppressed with cyclosporine $A$ : results of an international workshop. Clin Nephrol 24: 107-119.

21. Farnsworth A, Hall BM, Ng ABP, Duggin GG, Horvath JS, et al. (1984) Renal biopsy morphology in renal transplantation. A comparative study of the lightmicroscopic appearances of biopsies from patients treated with cyclosporin A or azathioprine prednisone and antilymphocyte globulin. Am J Surg Pathol 8 : 243-252.

22. Kazi JI, Furness PN, Nicholson M (1998) Diagnosis of early acute renal allograft rejection by evaluation of multiple histological features using a Bayesian Belief Network. J Clin Pathol 51: 108-113.

23. Kazi JI, Furness PN, Nicholson M, Ahmed E, Akhtar E, et al. (1999) Interinstitutional variation in the performances of Bayseian Belief Network for the diagnosis of acute renal graft rejection. Transplant Proc 31: 3152.

24. Jain S, Curwood V, Kazi JI, White SA, Furness PN, et al. (2000) Acute rejection in protocol renal transplant biopsies-institutional variations. Transplant Proc 32: 616.
25. Furness PN, Levesley J, Luo Z, Taub N, Kazi Jl, et al. (1999) A neura network approach to the diagnosis of early acute renal transplant rejection. Histopathology 35: 461-467.

26. Seron D, Anaya F, Marcen R, Moral RG, Martul EV, et al. (2008) Guidelines for indicating, obtaining, processing, and evaluating kidney transplant biopsies. Nefrologia 4: 385-396.

27. Racusen LC, Solez K, Colvin RB, Bonsib SM, Castro MC, et al. (1999) The Banff 97 working classification of renal allograft pathology. Kidney Int 55: 713723.

28. Mauiyyedi S, Crespo M, Collins AB, Schneeberger EE, Pascual MA, et al. (2002) Acute humoral rejection in kidney transplantation: II. Morphology, immunopathology, and pathologic classification. J Am Soc Nephrol 13: 779 787.

29. Racusen LC, Colvin RB, Solez K, Mihatsch MJ, Halloran PF, et al. (2003) Antibody mediated rejection criteria-An addition to the Banff ' 97 classification of renal allograft rejection. Am J Transplant 3: 708-714.

30. Cornell LD, Colvin RB (2005) Chronic allograft nephropathy. Curr Opin Nephro Hypertens 14: 229-234

31. Nankivell BJ, Borrows RJ, Fung CL, O'Connel PJ, Allen RD, et al. (2003) The natural history of chronic allograft nephropathy. N Eng J Med 349: 2326-2333.

32. Solez K, Colvin RB, Racusen LC, Siss B, Halloran PF, et al. (2007) Banff '05 meeting report: differential diagnosis of chronic allograft injury and elimination of chronic allograft nephropathy (CAN). Am J Transplant 7: 518-526.

33. Oguz Y, Doganci L, Bulucu F, Can C, Oktenli C, et al. (2002) Acute pyelonephritis causing acute renal allograft dysfunction. Int Urol Nephrol 34: 299-301.

34. Nickeleit V, Hirsch HH, Binet IF, et al. (1999) Polyomavirus infection of renal allograft recipients: From latent infection to manifest disease. J Am Soc Nephro 10: 1080-1089.

35. Hariharan S (2000) Recurrent and de novo diseases after renal transplantation Semin Dial 13: 195-199. 